\title{
A Dominant Mutation in Rpe65, D477G, Delays Dark Adaptation and Disturbs the Visual Cycle in the Mutant Knock-In Mice
}

\author{
Younghwa Shin, Gennadiy Moiseyev, Dibyendu Chakraborty, and Jian-xing Ma
}

From the Department of Physiology, University of Oklahoma Health Sciences Center, Oklahoma City, Oklahoma

\author{
Accepted for publication \\ November 3, 2016. \\ Address correspondence to \\ Jian-xing Ma, M.D., Ph.D., \\ Department of Physiology, \\ University of Oklahoma Health \\ Sciences Center, 941 Stanton L. \\ Young Blvd, BSEB Room \\ 328b, Oklahoma City, OK \\ 73104-5019. E-mail: jian-xing- \\ ma@ouhsc.edu.
}

\begin{abstract}
RPE65 is an indispensable component of the retinoid visual cycle in vertebrates, through which the visual chromophore 11-cis-retinal (11-cis-RAL) is generated to maintain normal vision. Various blinding conditions in humans, such as Leber congenital amaurosis and retinitis pigmentosa (RP), are attributed to either homozygous or compound heterozygous mutations in RPE65. Herein, we investigated $D 477 G$ missense mutation, an unprecedented dominant-acting mutation of RPE65 identified in patients with autosomal dominant RP. We generated a D477G knock-in (KI) mouse and characterized its phenotypes. Although RPE65 protein levels were decreased in heterozygous KI mice, their scotopic, maximal, and photopic electroretinography responses were comparable to those of wild-type (WT) mice in stationary condition. As shown by high-performance liquid chromatography analysis, levels of 11-cis-RAL in fully dark-adapted heterozygous KI mice were similar to that in WT mice. However, kinetics of 11-cis-RAL regeneration after light exposure were significantly slower in heterozygous KI mice compared with WT and RPE65 heterozygous knockout mice. Furthermore, heterozygous KI mice exhibited lower A-wave recovery compared with WT mice after photobleaching, suggesting a delayed dark adaptation. Taken together, these observations suggest that D477G acts as a dominant-negative mutant of RPE65 that delays chromophore regeneration. The KI mice provide a useful model for further understanding of the pathogenesis of RP associated with this RPE65 mutant and for the development of therapeutic strategies. (Am J Pathol 2017, 187: 517-527; http:// dx.doi.org/10.1016/j.ajpath.2016.11.004)
\end{abstract}

Vitamin A metabolism in vertebrate eyes, known as the retinoid visual cycle, plays a critical role in supporting visual function by generating the photosensitive chromophore 11-cis-retinal (11-cis-RAL) required to detect light. ${ }^{1}$ 11-cis-RAL binds with opsin to form rhodopsin in the outer segments of rod photoreceptors. On absorption of light, 11-cis-RAL isomerizes to photo-insensitive all-transretinal, inducing a conformational change in opsin (ie, metarhodopsin II) that subsequently triggers the downstream phototransduction pathway for visual sensation. ${ }^{2,3}$ However, rhodopsin kinase and arrestin quickly deactivate metarhodopsin II, and all-trans-retinal is dissociated from opsin as a result. Because rhodopsin must be regenerated for further phototransduction to occur, efficient regeneration of 11-cis-RAL via the retinoid visual cycle is essential for proper visual function.
The retinoid visual cycle is composed of numerous proteins located in the retinal pigment epithelium (RPE), interphotoreceptor matrix, and photoreceptors. ${ }^{4-7}$ All-trans-retinal dissociated from opsin is first reduced to all-trans-retinol by all-trans-retinal dehydrogenases in the outer segment of photoreceptors. Subsequently, all-transretinol binds to interphotoreceptor retinoid-binding protein that facilitates its transfer to the RPE. ${ }^{6,8,9}$ Within the RPE, all-trans-retinol is esterified to all-trans-retinyl ester (all-trans-RE) by lecithin/retinol acyl transferase. The retinoid isomerase RPE65 then converts all-trans-RE into

Supported by NIH grants EY012231, EY018659, EY019309, EY016507, and GM104934 (J.-x.M.); and Oklahoma Center for the Advancement of Science and Technology grant HR-13-076 (J.-x.M.).

Disclosures: None declared. 
11-cis-retinol. ${ }^{4,10-14}$ As the last step of the retinoid visual cycle, 11-cis-retinol is oxidized to 11-cis-RAL by 11-cisretinol dehydrogenases, and transported to photoreceptors where it recombines with opsin, generating rhodopsin. ${ }^{15-17}$

The retinoid visual cycle is frequently compromised because of genetic mutations in the genes involved in the visual cycle, leading to a variety of retinal disorders, such as fundus albipunctatus, Leber congenital amaurosis (LCA), and retinitis pigmentosa (RP) ${ }^{18-22}$ In fact, a key enzyme of the retinoid visual cycle, RPE65, alone has $>100$ mutations reported to associate with LCA type 2 (LCA2) and autosomal recessive RP. ${ }^{23}$ The phenotype most commonly observed in patients with mutations in RPE65 is LCA2 or severe earlyonset retinal degeneration, which causes visual impairment from birth and/or progression to complete blindness by the third decade of life. ${ }^{24}$ Up to $16 \%$ of all diagnosed LCAs are attributed to mutations in RPE65 at the LCA2 locus (1p31), and patients with LCA2 carry mutations in both copies of RPE65, either as homozygous or compound heterozygous. ${ }^{25}$

In 2011, an unprecedented dominant mutation of RPE65 was identified from a large Irish family with autosomal dominant RP (adRP) and a small Irish family with a choroideremia. ${ }^{26} \mathrm{~A}$ single-nucleotide substitution (c.1430G $>$ A) changed codon 477 from Asp to Gly (D477G), and the patients carrying a single copy of such mutated RPE65 exhibited varying degrees of visual defects that are generally less aggressive compared to what are commonly observed in recessive RPE65 cases. Another group has recently reported an independent study on patients with $D 477 G$ who exhibited similar clinical features, and also mentioned a possible association of $D 477 G$ with adultonset vitelliform macular dystrophy. ${ }^{27}$

The purpose of this study was to generate a heterozygous $D 477 G$ knock-in (KI) mouse as a model for investigation of adRP associated with this dominant-negative mutation of RPE65. The present article details characterization of the $D 477 G$ KI model by examining retinal morphology, the retinoid visual cycle, and visual function.

\section{Materials and Methods}

\section{Generation of D477G KI Mice}

A targeting vector was generated from a BAC clone spanning a 7.4-kb region including exon 13 , where the point mutation was introduced. The construct containing the point mutation and FRT-flanked neomycin cassette was then subcloned into the pSP72 backbone vector for retransformation before electroporation. NotI linearized targeting construct was then electroporated into embryonic stem cells $(129 / \mathrm{SvEv} \times \mathrm{C} 57 \mathrm{~B} 1 / 6$ hybrid). Positive clones were selected by G418 resistance, followed by expansion for screening of homologous recombination by genomic PCR (LAN1 forward, 5' CCAGAGGCCACTTGTGTAGC-3'; and A2 reverse, 5'-GGAACCACTTGTCCAACTCAG-3') and Southern blotting Sca1 or Mfe1 digested DNA with a probe (iNeo) targeted against the Neo cassette. Validated clones were then injected into blastocysts to generate a chimera line, which subsequently crossed with FLP mice (InGenious Targeting Laboratory, Ronkonkoma, NY) to generate Neo deleted heterozygous mice. Correct excision of the selectable marker was confirmed by PCR strategy targeting the DNA region that differentiates wild-type (WT) allele (448 bp) and FRT-deleted KI allele (549 bp). Primer sequences are as follows: D477G forward 1, 5'-TGGAATCAATGAGTGTCTGGACT-3'; and D477G reverse 1, 5'AAGAACCCTAGCCACAGCATA-3' . Rpe65 knockout (KO) mice were kindly provided by Dr. Michael Redmond (National Eye Institute, Bethesda, MD). ${ }^{12}$ Heterozygous KO mice were obtained by crossing Rpe65 KO mice with WT mice.

\section{Animals}

All animal studies were approved by the Institutional Animal Care and Use Committee of the University of Oklahoma Health Sciences Center (Oklahoma City, OK), and performed following the guidelines of the Association for Research in Vision and Ophthalmology statement for the Use of Animals in Ophthalmic and Vision Research (http://www.arvo.org/about_arvo/policies/statement_for_ the_use_of_animals_in_ophthalmic_and_visual_research). The animals were maintained in standard housing with 12-hour light/dark cycles and food and water ad libitum.

\section{Real-Time PCR}

Levels of the RPE65 mRNA were examined by real-time PCR. Enucleated eyes were carefully dissected and immediately snap frozen in liquid nitrogen. Total RNA was isolated by Trizol and chloroform, and $0.5 \mu \mathrm{g}$ RNA was reverse transcribed to cDNA (Applied Biosystems, Foster City, CA). The generated cDNA was then used as template for real-time PCR with a primer pair specific for the mouse RPE65 mRNA. Gadph was used as a housekeeping gene for normalization ( $n=3$ per group).

\section{Western Blot Analysis}

Enucleated eyes from WT, heterozygous KI, and homozygous KI mice were dissected to obtain the eyecup containing the RPE, choroid, and sclera. The eyecups were homogenized by sonication in phosphate-buffered saline, followed by centrifugation at $16,000 \times g$ for 15 minutes. Total protein $(25 \mu \mathrm{g})$ from the collected supernatant was resolved on $10 \%$ SDS-PAGE gel. RPE65 was detected by a rabbit polyclonal antibody against RPE65 (1:1000), as previously described..$^{5} \beta$-Actin was detected by anti- $\beta$-actin-horseradish peroxidase (SC1616HRP, 1:2000; Santa Cruz Biotechnology, Dallas, TX). Band densitometry was performed with ImageJ software version 1.50i (NIH, Bethesda, MD; http://imagej.nih.gov/ij).

\section{Light and Electron Microscopy}

Tissues were collected, processed, and embedded in plastic, as described elsewhere. ${ }^{28,29}$ For light microscopy, plastic 
sections $(0.75 \mu \mathrm{m}$ thick $)$ were imaged with Zeiss Observer Z1 (Zeiss, Thornwood, NY). For transmission electron microscopy, thin (600 to $800 \AA$ ) sections were collected on copper $75 / 300$ mesh grids and stained with $2 \%(w / v)$ uranyl acetate and Reynold's lead citrate. JEOL 100CX electron microscope (JEOL, Akishima, Tokyo) was used to view sections, at an accelerating voltage of $60 \mathrm{kV}$.

\section{Immunohistochemistry}

Enucleated eyes were briefly fixed in $4 \%$ paraformaldehyde, followed by a needle puncture just below the limbus and additional fixation in $4 \%$ paraformaldehyde for 30 minutes. The anterior portion of the eyes containing the cornea and lens was then dissected away, and remaining eyecups were cryoprotected in graded sucrose solutions $(10 \%$ to $30 \%$ in phosphate-buffered saline). The eyecups were embedded in OCT compound and frozen over liquid nitrogen. Cryosections ( $12 \mu \mathrm{m}$ thick) of the retinas were then incubated in blocking solution (5\% bovine serum albumin plus $0.3 \%$ Triton X-100 in phosphate-buffered saline), followed by overnight incubation at $4{ }^{\circ} \mathrm{C}$ with primary antibody diluted in blocking solution. The sections were washed three times with phosphate-buffered saline containing $0.3 \%$ Triton X-100, and incubated with appropriate fluorophore-conjugated secondary antibodies for 1 hour at room temperature. The following antibodies were used: S-opsin (ab1778, 1:200; Millipore, Temecula, CA), M-opsin (ab5405, 1:200; Millipore), rhodopsin (1D4, 1:500), and RPE65 (PETLET, 1:200).

\section{Isomerase Activity Assay}

Isomerase activity assay was performed, as previously described, ${ }^{30}$ in a dark room under red light (catalog number A19/R/LED; Feit Electric, Pico Riverea, CA). In brief, the eyecups were homogenized with a glass grinder in a buffer containing $10 \mathrm{mmol} / \mathrm{L}$ bis-Tris-propane $(\mathrm{pH} \mathrm{8.0)}$ and 100 $\mathrm{mmol} / \mathrm{L} \mathrm{NaCl}$. All-trans-[11, $\left.12-{ }^{3} \mathrm{H}\right]$-retinol in ethanol $(1 \mathrm{mCi} / \mathrm{mL}, 52 \mathrm{Ci} / \mathrm{Mmol} ; 1 \mathrm{Ci}=37 \mathrm{GBq}$; PerkinElmer, Waltham, MA) was dried under argon and resuspended in the same volume of dimethylformamide. Subsequently, $2 \mu \mathrm{L}$ of all-trans-[11, 12- $\left.{ }^{3} \mathrm{H}\right]$-retinol in dimethylformamide and $250 \mu \mathrm{g}$ of total proteins were added into $200 \mu \mathrm{L}$ of the reaction buffer $(10 \mathrm{mmol} / \mathrm{L}$ bis-Tris-propane, $\mathrm{pH}$ 8.0/100 $\mathrm{mmol} / \mathrm{L} \mathrm{NaCl}$ ), including $0.5 \%$ bovine serum albumin and $25 \mu \mathrm{mol} / \mathrm{L}$ cellular retinaldehyde-binding protein. After a 2hour incubation period, generated retinoids were extracted by methanol/hexane, and the upper organic phase was analyzed by normal-phase high-performance liquid chromatography (HPLC) coupled to Radiomatic 610TR Flow Scintillation Analyzer (PerkinElmer).

\section{HPLC Retinoid Profiling}

Dark-adapted mice were sacrificed, and their eyes enucleated ( $n=4$ per group). The whole eyes were homogenized with a glass grinder in lysis buffer $\left[10 \mathrm{mmol} / \mathrm{L} \mathrm{NH}_{2} \mathrm{OH}, 50 \%\right.$ ethanol,
$50 \%$ 2-(N-morpholino)ethanesulfonic acid, $\mathrm{pH}$ 6.5], and retinoids were extracted with hexane. Solvent was evaporated under argon gas, and dried retinoid samples were resuspended in $200 \mu \mathrm{L}$ of HPLC mobile phase (11.2\% ethyl acetate, $2.0 \%$ dioxane, $1.4 \%$ octanol, and $85.4 \%$ hexane) and injected into the HPLC machine (515 HPLC pump; Waters Corp., Milford, MA) with a normal phase Lichrosphere SI-60 (Alltech, Deerfield, IL) $5-\mu \mathrm{m}$ column and isocratic mobile phase $(1 \mathrm{~mL} /$ minute). The HPLC retinoid profiling was performed following a previous protocol ${ }^{10}$ in a dark room under red light.

\section{Measurement of 11-cis-Retinal Recovery by HPLC}

Mice were dark adapted overnight before experiment day ( $n=4$ per group). On the day of experiment, mice were subjected to 5000 lux light for 30 minutes. Immediately after the 30 minutes of light exposure, mice were returned to dark, where they recovered for 15 or 30 minutes. After the recovery, mice were sacrificed and their eyes enucleated. Collected eyes were processed for HPLC retinoid profiling, as described above.

\section{ERG Data}

Mice were dark adapted overnight before performing electroretinography (ERG). Mice were then anesthetized by an i.p. injection using $3 \mu \mathrm{L} / \mathrm{g}$ body weight of $40 \mathrm{mg} / \mathrm{mL}$ ketamine and $3 \mathrm{mg} / \mathrm{mL}$ xylazine diluted with saline. Pupils were dilated with $1 \%$ cyclopentolate hydrochloride ophthalmic solution (Cyclogyl, Fort Worth, TX) and 10\% phenylephrine-HCl. Hypromellose ophthalmic demulcent solution (2.5\%; Gonak, Lake Forest, IL) was applied to each cornea, followed by the placement of gold wire electrodes. Stainless steel electrodes were placed into the right cheek and the tail to serve as a reference and the ground, respectively. Espion $E^{3}$ system with a Ganzfeld ColorDome system was used to perform ERG procedures (Diagnosys LLC, Lowell, MA). The intensities used for scotopic, maximal, and photopic ERG responses are $0.8,40$, and $40 \mathrm{~cd} \cdot \mathrm{s} / \mathrm{m}^{2}$, with $10 \mathrm{~cd} / \mathrm{m}^{2}$ as background, respectively (WT/WT, $n=12$; and WT/KI, $n=8$ ). For double-flash ERG, fully dark-adapted mice were subjected to the first flash (ie, probe flash, $4 \mathrm{~cd} \cdot \mathrm{s} / \mathrm{m}^{2}$ ), followed by the second flash (ie, test flash, $40 \mathrm{~cd} \cdot \mathrm{s} / \mathrm{m}^{2}$ ) at various delay times ranging from 0.2 to 1.5 seconds (WT/WT, $n=6$; and WT/KI, $n=8)$. For dark-adaptation recovery, responses of the fully dark-adapted mice to single flash $\left(10 \mathrm{~cd} \cdot \mathrm{s} / \mathrm{m}^{2}\right)$ stimulus were acquired before and after intense bleaching under $1000 \mathrm{~cd} / \mathrm{m}^{2}$ for 2 minutes. Mice were allowed to recover in the dark up to 60 minutes while acquiring single-flash ERG $\left(10 \mathrm{~cd} \cdot \mathrm{s} / \mathrm{m}^{2}\right)$ every 5 minutes (WT/WT, $n=10$; and WT/KI, $n=16$ ).

\section{Results}

\section{Generation of the D477G KI Mouse}

We have generated a KI mouse model harboring the missense mutation at c.1430G >A (Asp477Gly), as a model 
for human adRP patients with the $D 477 G$ mutation (Figure 1A). A 13.4-kb targeting vector was generated that includes exons 11 through 14, with the mutant codon 477 in exon 13 (c.1430G $>$ A, p.D477G) and FRT-flanked Neo cassette downstream of exon 13 (Figure 1A). The linearized targeting construct was electroporated into embryonic stem cells $(129 / \mathrm{SvEv} \times \mathrm{C} 57 \mathrm{Bl} / 6 \mathrm{~J}$ hybrid $)$ for a site-specific homologous recombination, and the positive clones with correctly targeted allele were identified and confirmed by PCR and Southern blotting (Figure 1, B and C). Germline competent chimeric mice were generated from those clones, and were subsequently crossed with FLP mice to excise the FRT-flanked Neo cassette. A PCR strategy was designed (forward 1, 5'-TGGAATCAATGAGTGTCTGGACT-3'; and reverse $1,5^{\prime}$-TATGCTGTGGCTAGGGTTCTT-3') to distinguish the WT allele (448 bp) from the mutant allele
(549 bp after the Neo cassette deletion) (Figure 1D). An additional PCR was performed with a primer pair (RPE65 forward, 5'-CACTGTGGTCTCTGCTATCTTC-3'; and RPE65 reverse, 5'-GGTGCAGTTCCATTCAGTT-3') to amplify the region overlapping the point mutation, and the resulting amplicon was sequence confirmed for the presence of the mutant codon (Figure 1E). The KI line was then propagated by intercrossing the heterozygous KI mice, to generate littermate WT control and homozygous KI mice.

Expression Levels of the RPE65 mRNA and Protein in D477G KI Mice

Expression of RPE65 was examined at both the transcriptional and translational levels. RPE65 mRNA levels were comparable among WT, heterozygous $D 477 G \mathrm{KI}$, and homozygous
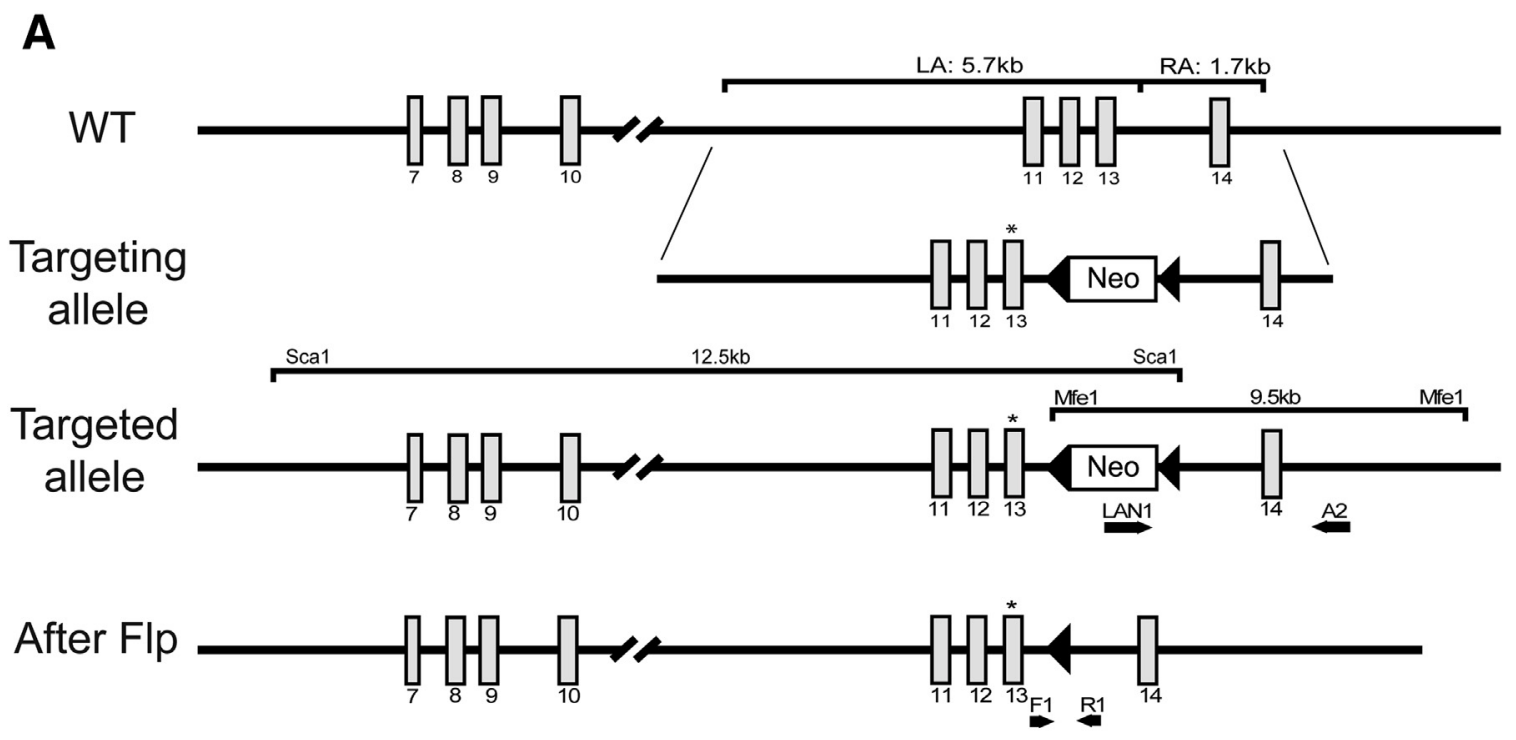

B

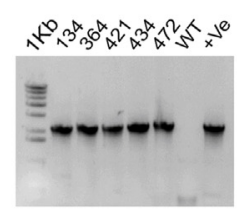

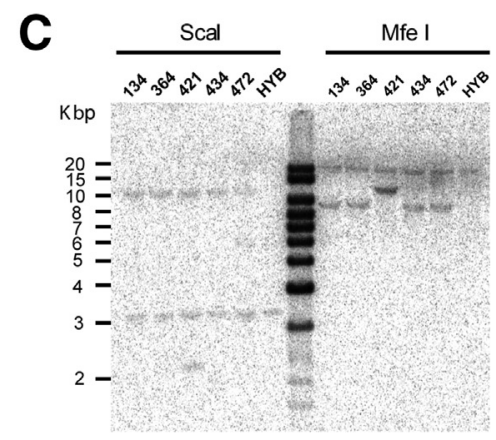

Figure 1 Generation of the D477G KI mouse model. A: The D477G targeting strategy is shown as a schematic representation. The targeting construct is designed to span $5.7 \mathrm{~kb}$ upstream of the point mutation (LA) and $1.7 \mathrm{~kb}$ downstream of the Neo cassette (RA). The Neo cassette was inserted in the intron region between exons 13 and 14. FRT-flanked Neo cassette in the targeted allele is deleted by FRT-FLP recombination, thereby generating a mutant allele containing the point mutation in exon 13 (asterisks). B: A PCR strategy was used to screen for the positive clones. LAN1 and A2 primer pair targeting the RA yielded a 2.18-kb product. WT genomic DNA was used as a negative control, and an individual clone (before reconfirmation) was used as a positive control. C: The PCR-confirmed clones were further validated by Southern blotting. DNA digested with ScaI or MfeI was hybridized with a probe against the Neo cassette. Corresponding signals from ScaI and MfeI were detected at 12.5 and $9 \mathrm{~kb}$, respectively. D: Genotyping PCR using primers F1 and R1 flanking the FRT site after Flp-mediated deletion of the Neo cassette produced 549- and 448-bp products, each representing targeted and WT allele, respectively. Extra 101 bp present in targeted allele comprises 34-bp-long FRT sequence and 67-bp-long remnants from the Neo cassette. E: Sequence confirmation of the point mutation at codon 477. c.1430G $>A$ is confirmed by sequencing the $P C R$ amplicon obtained with a primer pair encompassing the region of the point mutation. $+V e$, positive; F, forward; HET, heterozygous; HYB, hybrid; KI, knock in; LA, left homology arm; R, reverse; RA, right homology arm; WT, wild type. 


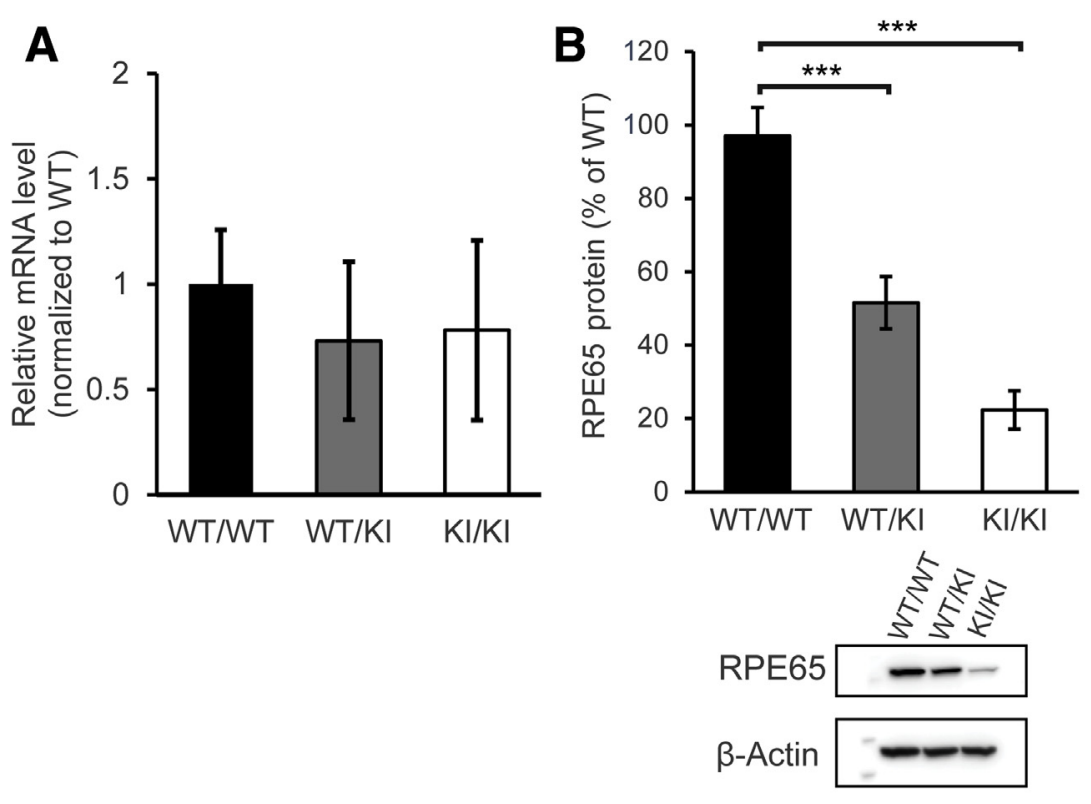

Figure 2 Reduced RPE65 levels in D477G KI mice. A: Relative levels of the RPE65 mRNA in the eyecups of these mice were analyzed by real-time $P C R$. Representative values are normalized to mRNA levels of a housekeeping gene (Gapdh). B: Eyecups containing sclera, choroid, and RPE were dissected and homogenized. The eyecup homogenates from wild-type (WT/WT), the heterozygous $\mathrm{KI}(\mathrm{WT} / \mathrm{KI})$, and the homozygous KI (KI/KI) mice were then subjected to Western blot analysis with an antibody against RPE65 (PETLET). $\beta$-Actin was used as loading control. Densitometry was used for semiquantification. C: Immunofluorescence labeling of RPE65 on the retinal cross sections. RPE65 is shown in red, and nucleus is shown in blue (DAPI). Error bars represent SDs from the triplicates within the experiment (A). $n=3$ per group (A); $n=4$ per group (B). ${ }^{* * * P}<0.001$ (t-test). Scale bar $=$ $50 \mu \mathrm{m}$ (C). GCL, ganglion cell layer; INL, inner nuclear layer; IPL, inner plexiform layer; KI, knock in; $\mathrm{ONL}$, outer nuclear layer; $\mathrm{OPL}$, outer plexiform layer; $\mathrm{OS}$, outer segment; RPE, retinal pigment epithelium; WT, wild type.
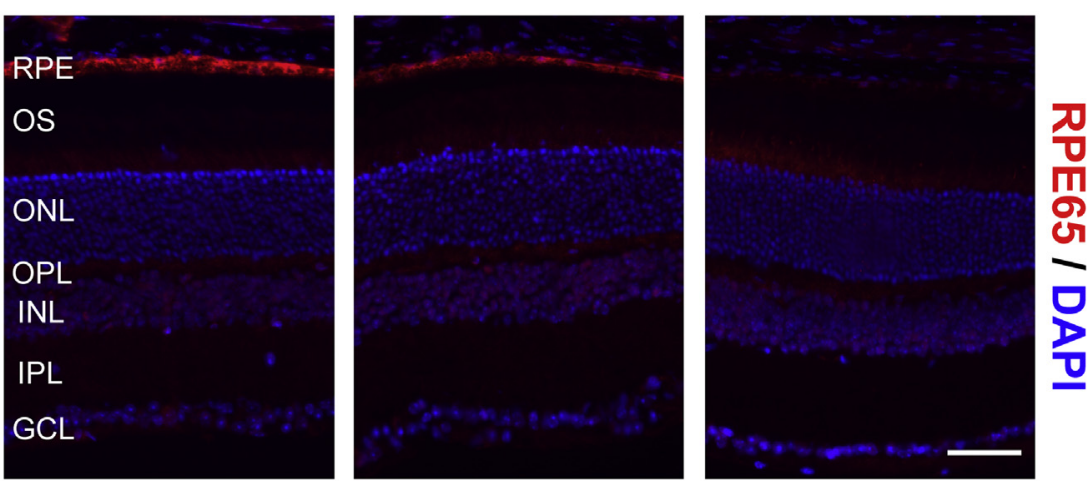

KI mice (Figure 2A). However, levels of the RPE65 protein were reduced in the heterozygous KI mice (approximately $51 \%$ of WT/WT) and even lower in the homozygous KI mice (approximately 22\% of WT) (Figure 2B). Similarly, immunofluorescence labeling of RPE65 on the ocular cross sections from the heterozygous KI and the homozygous KI mice showed lower fluorescence intensities in the RPE, corresponding to reduced RPE65 expression, as shown by the Western blotting result (Figure 2C). This finding resonates with a previous report that predicted destabilization of RPE65 protein because of increased folding free energy ${ }^{26}$ Other than a reduction in protein levels, RPE65 was detected in the RPE layer in all three groups (Figure 2C).

\section{No Change in Retinal Morphology and 0psin Expression in D477G KI Mice}

Numerous studies indicate that photoreceptor survivalparticularly cone photoreceptors - is sensitive to changes in
RPE65 expression. ${ }^{31}$ Therefore, we sought to examine whether heterozygous $D 477 G$ KI exhibited any signs of anomaly in the retinal morphology. However, the retinal cross sections immunolabeled with antibodies for S-opsin, M-opsin, and rhodopsin showed no conspicuous signs of abnormalities in 3.5-month-old WT and the heterozygous KI mice. Thickness of the outer nuclear layer and inner nuclear layer also appeared to be comparable between WT and the heterozygous KI mice (Figure 3A). Because human patients with $D 477 G$ have a wide range of onset, we wanted to examine whether our KI mice develop retinal phenotypes at a much later stage as well. However, the retinal cross sections from 13-month-old WT and heterozygous KI mice did not present any signs of retinal degeneration (Figure 3B). Transmission electron microscopy images revealed an unusual accumulation of lipid droplet-like organelles in the RPE of the heterozygous KI mice (Figur 3C). However, further investigation is required to determine their physiological significance. 
A
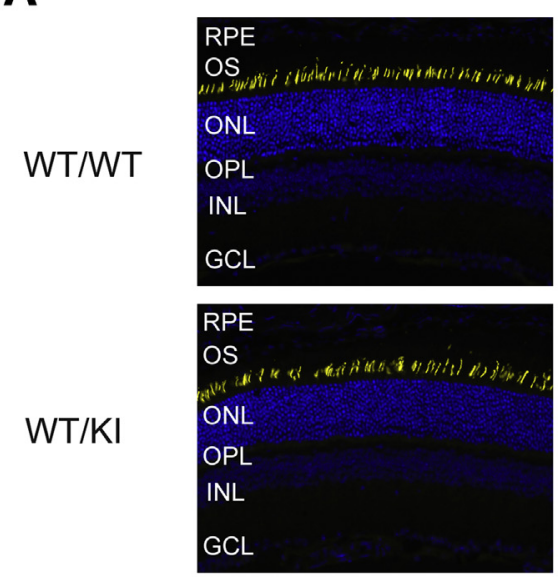

M-Opsin
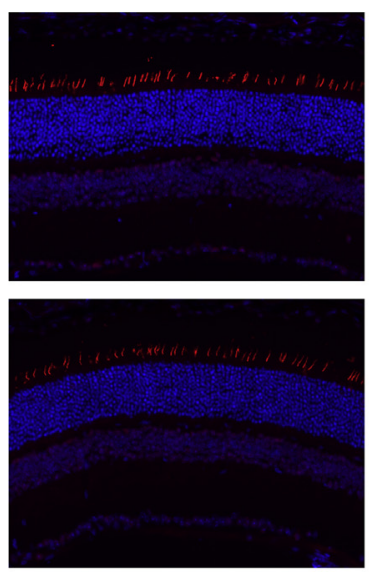

Rhodopsin

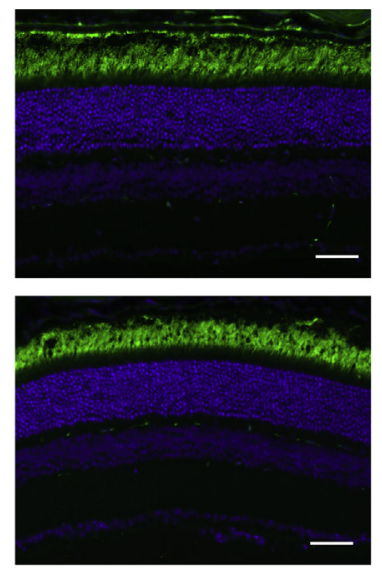

B
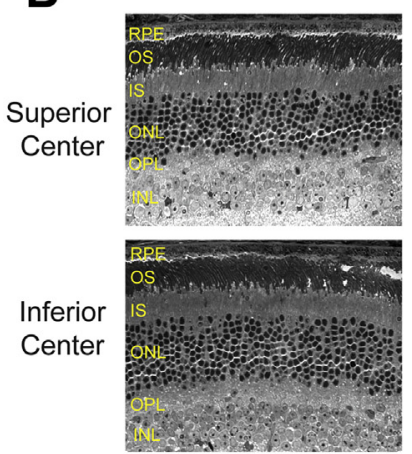

WT/KI

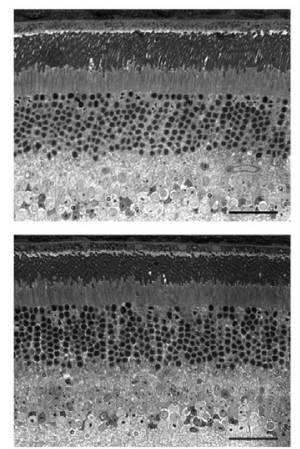

C
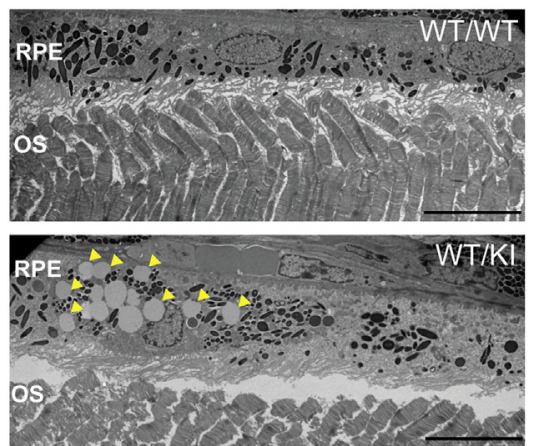

Figure 3 Analysis of opsin expression and localization. A: Retinal cross sections from 3.5-month-old WT/WT and WT/KI mice were immunolabeled with antibodies against short-wavelength cone opsin (S-opsin, yellow), midwavelength cone opsin (M-opsin, red), or rhodopsin (green). Nuclei were counterstained with DAPI (blue). B: Light microscopic images of the retinal cross sections from 13-month-old WT/WT and WT/KI mice. C: Transmission electron microscopy images of 13-month-old WT/WT and WT/KI. An unusual accumulation of oil droplet-like organelles in the RPE of WT/KI mice are indicated by arrowheads. Scale bars: $50 \mu \mathrm{m}(\mathbf{A}) ; 40 \mu \mathrm{m}(\mathbf{B}) ; 10 \mu \mathrm{m}(\mathbf{C})$. GCL, ganglion cell layer; INL, inner nuclear layer; IS, inner segment; KI, knock in; 0NL, outer nuclear layer; $\mathrm{OPL}$, outer plexiform layer; $\mathrm{SS}$, outer segment; RPE, retinal pigment epithelium; WT, wild type.

\section{Heterozygous D477G KI Mice Retain Normal Visual Function}

With respect to normal retinal morphology observed, we then examined the visual function of the heterozygous KI mice at various ages ( 3 to 5 months). First of all, fully dark-adapted mice were subjected to dim flash light $\left(0.8 \mathrm{~cd} \cdot \mathrm{s} / \mathrm{m}^{2}\right)$ to test rod-driven visual function (Figure $\left.4 \mathrm{~A}\right)$. As expected, scotopic vision was normal up to 5 months of age. Using a more intense light stimulus $\left(40 \mathrm{~cd} \cdot \mathrm{s} / \mathrm{m}^{2}\right)$, visual function driven by a combination of both the rod and cone pathways was assessed (Figure 4B). Maximal response was also normal up to 5 months of age. Last, isolated cone function was examined by $40 \mathrm{~cd} \cdot \mathrm{s} / \mathrm{m}^{2}$ stimuli with a background of $10 \mathrm{~cd} / \mathrm{m}^{2}$ (Figure 4C). Similarly, photopic response was also normal up to 5 months of age. This finding is reminiscent of animal models that exhibited normal visual function despite reduced levels of RPE $65^{32}$ or the absence of other visual cycle enzymes, such as RDH5. ${ }^{17}$

\section{Reduction in Isomerase Activity in Heterozygous D477G KI Mice}

Studies on Rpe65 ${ }^{-1-}$ mice have shown that complete lack of RPE65 leads to the absence of detectable 11-cis-RAL in the RPE, as well as significantly increased levels of all-transRE. ${ }^{12}$ Several reports on recessive mutants of RPE65 using KI models have reported similar observations on altered retinoid profiles that are attributed to decreased RPE65 expression. ${ }^{32,33}$ Therefore, we expected to observe an altered retinoid profile in the RPE of $D 477 G \mathrm{KI}$ mice. Retinoid profile analysis using HPLC on the fully dark-adapted eyeball samples showed that levels of 11-cis-RAL were comparable between WT and the heterozygous KI mice (Figure 5A). This finding is in accordance with normal visual function demonstrated by ERG (Figure 4). Unlike what we expected, levels of all-trans-RE in the heterozygous KI mice were also comparable to WT mice (Figure 5A).

Because computer modeling of the D477G mutant predicted substantial structural changes to the RPE65 protein 


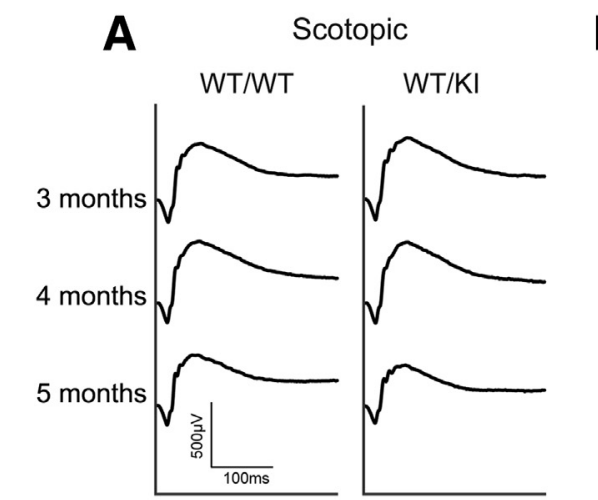

B

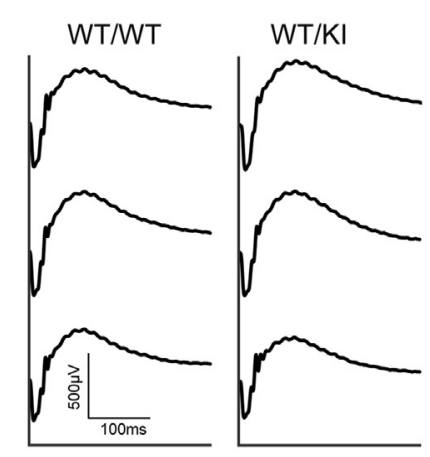

C Photopic
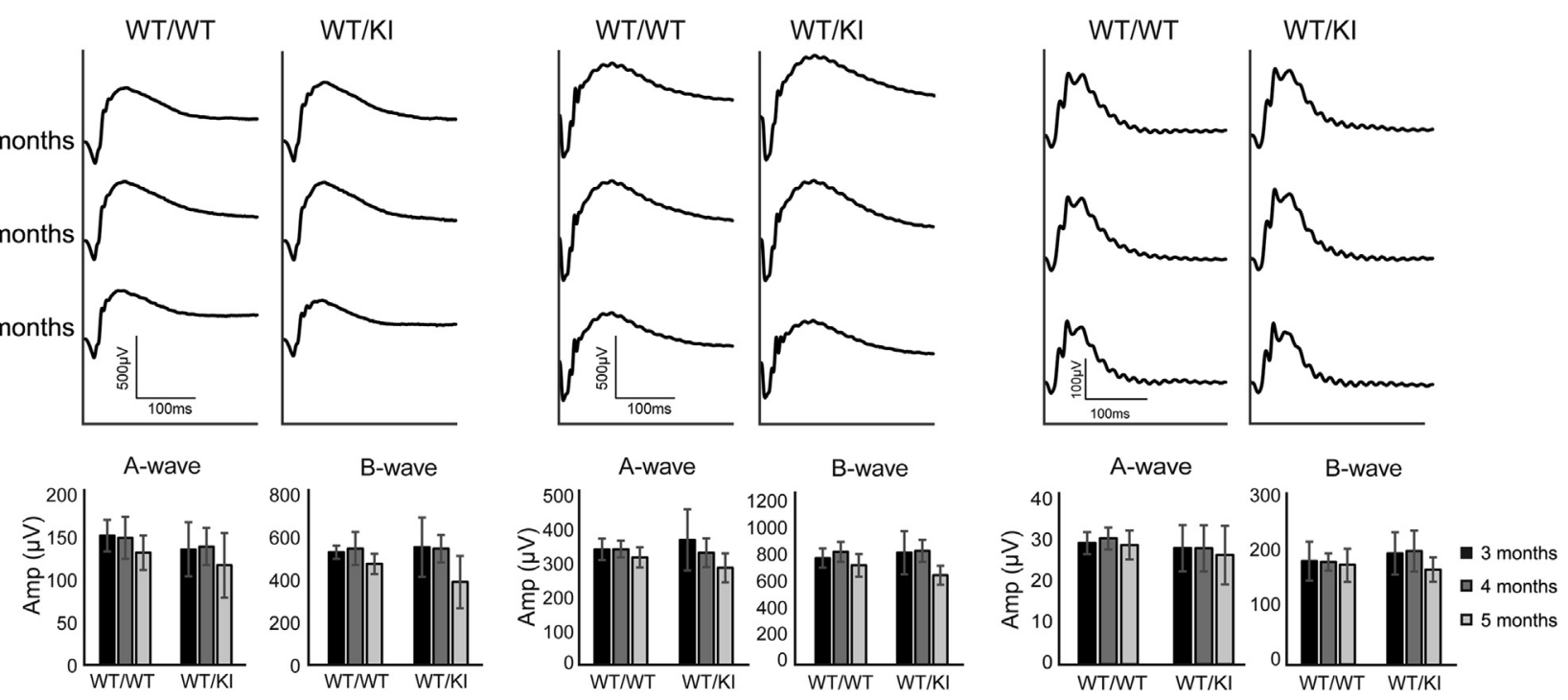

Figure 4 Visual function assessment by electroretinography (ERG): Mice were fully dark adapted overnight before ERG. A: Scotopic ERG measurement $\left(0.8 \mathrm{~cd} \cdot \mathrm{s} / \mathrm{m}^{2}\right)$ on WT/WT and WT/KI mice at 3, 4, and 5 months of age. B: Maximal ERG response $\left(40 \mathrm{~cd} \cdot \mathrm{s} / \mathrm{m}^{2}\right)$ of these mice. C: Photopic ERG measurements $\left(40 \mathrm{~cd} \cdot \mathrm{s} / \mathrm{m}^{2}\right.$ with background of $\left.10 \mathrm{~cd} / \mathrm{m}^{2}\right)$ of these mice. Top panels indicate averaged waveforms from each genotype at indicated ages, and bottom panels are the corresponding histograms of A- and B-wave values. Data are given as means \pm SD (A-C, bottom panels). $n=12(\mathbf{A}-\mathbf{C}$, WT/WT); $n=8(\mathbf{A}-\mathbf{C}$, WT/KI). KI, knock in; WT, wild type.

(ie, turning of $\beta$ propeller VII) that could affect iron coordination required for the enzymatic activity, ${ }^{26}$ we sought to examine the enzymatic property of the mutant by measuring isomerase activity with the isolated eyecup samples. The isomerase activity assay showed that the heterozygous $\mathrm{KI}$ mouse eyecups produced significantly lower levels of 11-cis-retinol (approximately 79\% of WT, $P<0.05$ ) (Figure 5B), suggesting that the KI mice have reduced isomerase activities (Figure 5B).

\section{Delayed Dark Adaptation in Heterozygous D477G KI Mice}

Because human patients with $D 477 G$ are initially presented with delayed dark adaptation, we tested whether our KI mice can recapitulate such phenotypes. First, we used a double-flash ERG technique to investigate recovery function of rod photoreceptors. Fully dark-adapted mice were subjected to a single probe flash (DA, $\left.4 \mathrm{~cd} \cdot \mathrm{s} / \mathrm{m}^{2}\right)$, followed by the test flash at various times $\left(40 \mathrm{~cd} \cdot \mathrm{s} / \mathrm{m}^{2}\right)$ (Figure 6A). Response from each test flash was normalized by the fully dark-adapted A-wave amplitude. However, there was no significant difference between WT and the heterozygous KI mice, suggesting that $\mathrm{D} 477 \mathrm{G}$ does not affect rod functional recovery under a mild bleaching condition (Figure 6B). We then used a stronger bleaching condition, where fully dark-adapted mice were subjected to bleaching $\left(1000 \mathrm{~cd} / \mathrm{m}^{2}\right.$ for 2 minutes), and single-flash ERG responses to $10 \mathrm{~cd} \cdot \mathrm{s} / \mathrm{m}^{2}$ were recorded every 5 minutes while recovering in the dark for the next 60 minutes (Figure 6, C and D). Interestingly, the heterozygous
KI mice had a lower A-wave recovery compared to WT mice (Figure 6, C and D).

\section{Impaired Visual Chromophore Regeneration in Heterozygous D477G KI Mice}

Because impaired synthesis of 11-cis-RAL is known to cause delayed dark adaptation, we decided to examine whether the rate of 11-cis-RAL synthesis is affected in the presence of D477G. Mice were exposed to bleaching condition (5000 lux for 30 minutes), followed by dark adaptation for 15 and 30 minutes. Using HPLC retinoid profile analysis, we examined the retinoid contents from the eyeballs collected at 15 and 30 minutes of dark adaptation after the light exposure. As expected, levels of 11-cis-RAL after 15 minutes of dark adaptation were lower in the heterozygous KI mice and Rpe65 heterozygous KO mice, compared to WT (heterozygous KI, approximately $49 \%$ of WT; and heterozygous KO, approximately $72 \%$ of WT) (Figure 7A). Even after 30 minutes of dark adaptation, levels of 11-cisRAL were still lower in the KI and KO mice, compared to WT (heterozygous KI, approximately $52 \%$ of WT; and heterozygous KO, approximately 89\% of WT) (Figure 7A). The heterozygous KI mice contained significantly lower levels of 11-cis-RAL than heterozygous KO mice at both of the time points, suggesting that the presence of the $\mathrm{D} 477 \mathrm{G}$ mutant protein suppresses the enzymatic activity of WT RPE65. This notion was further supported by the corresponding increase in levels of all-trans-RE, the substrate of RPE65, in the heterozygous KI mice compared to WT and the heterozygous $\mathrm{KO}$ mice (Figure 7B). Taken together, our 

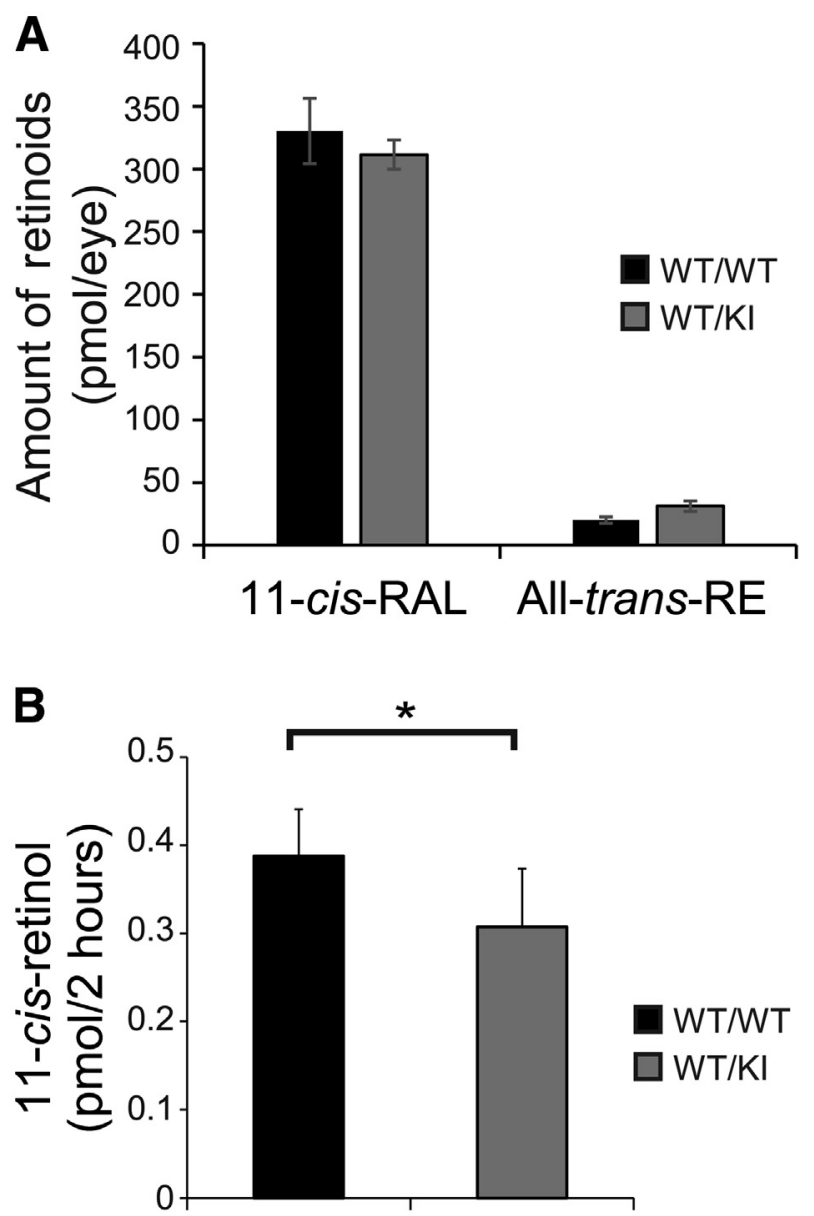

Figure 5 Retinoid profile and isomerase activity in D477G KI mice. A: High-performance liquid chromatography (HPLC) retinoid profile analysis on the fully dark-adapted eyeballs from WT/WT and WT/KI mice. Both 11cis-retinal (11-cis-RAL) and all-trans-retinyl ester (all-trans-RE) levels were measured. B: Isomerase assay using the eyecups from indicated mice. Levels of the generated 11-cis-retinol were measured by HPLC. The eyecup homogenates used for isomerase assay were examined by Western blot analysis to ensure the comparable amount of the total protein. Data are given as means \pm SEM (A and B). $n=4$ (A); $n=6$ (B). ${ }^{*} P<0.05$ ( $t$-test). KI, knock in; WT, wild type.

data suggest that delayed dark adaptation in the heterozygous KI is most likely because of the presence of the $D 477 G$ allele that hinders the efficiency of 11-cis-RAL synthesis.

\section{Discussion}

D477G is the only dominant mutant of RPE65 reported thus far, but the molecular mechanism behind the pathogenesis leading to vision impairment in humans remains elusive. ${ }^{26,27}$ Herein, we have generated a $D 477 G$ mutant KI mouse model to investigate the molecular event(s) underlying vision impairment caused by this dominant RPE65 mutant. The heterozygous KI mice showed delayed dark adaptation in visual sensitivity, recapitulating the phenotypes of patients with adRP associated with $D 477 G$. Furthermore, we have observed an unprecedented phenomenon that presence of the $\mathrm{D} 477 \mathrm{G}$ protein in the heterozygous KI mice reduced the regeneration of 11-cis-RAL and reduced isomerase activity, suggesting that D477G acts as a dominant-negative mutant and suppresses the isomerase activity of WT RPE65. This represents a pathogenic mechanism distinct from all recessive RPE65 mutations studied previously. As such, this poses a unique challenge to currently existing gene therapy for LCA2 because the current therapeutic strategy relies on restoring the 11-cis-RAL production by delivering functional copies of WT RPE65. ${ }^{24,34-36}$ Because $D 477 G$ is a dominant-negative mutant, future therapeutic development may have to consider a different approach, such as silencing of the mutant transcript or correcting the mutant codon via a genome editing technique, such as CRISPR.

Within the past decade, at least two recessive mutants of RPE65 (ie, R91W and P25L) have been characterized in KI mouse models that demonstrated delayed chromophore regeneration. ${ }^{32,33}$ The phenotypes, however, were detected only in homozygous KI mice because of the lack of WT RPE65. This is consistent with previous observations in humans (ie, a single copy of WT RPE65 is sufficient to support normal visual function, including normal darkadaptation recovery). ${ }^{37}$ In the present study, we demonstrated that one allele of $D 477 G$ in heterozygous KI mice resulted in delayed chromophore regeneration at both the biochemical and physiological levels. Our ERG data combined with HPLC analysis on the retinoid profile suggest that delayed chromophore regeneration is most likely because of impaired isomerase activity. To make an important distinction between a true mutant effect and haploinsufficiency (ie, decreased enzymatic activity because of less protein expression), our HPLC retinoid profile analysis included a side-by-side comparison between heterozygous $D 477 G \mathrm{KI}$ and heterozygous Rpe65 KO mice. Our data suggest that the chromophore regeneration at 15 minutes of dark adaptation is in fact slower in the heterozygous KI compared to the heterozygous $\mathrm{KO}$ mice. The difference between the heterozygous KI and the heterozygous $\mathrm{KO}$ mice persisted to 30 minutes of dark adaptation. Although the exact mechanism by which the D477G mutant protein disturbs WT RPE65 activity remains to be investigated, our observation indicates that the KI mouse model recapitulates the delayed dark adaptation symptom reported in human patients with the $D 477 G$ mutation. ${ }^{26}$

Despite the impaired chromophore regeneration and delayed dark adaptation, our KI mice lack other disease manifestations present in human patients (ie, changes in retinal morphology and visual function deficit). Considering a widely variable age of onset in human patients, it is plausible that the pathogenic mechanism of $\mathrm{D} 477 \mathrm{G}$ requires an additional component, such as an environmental stressor. For example, the husbandry environment where the ambient illuminance is $<100$ lux may not impose a heavy work load 


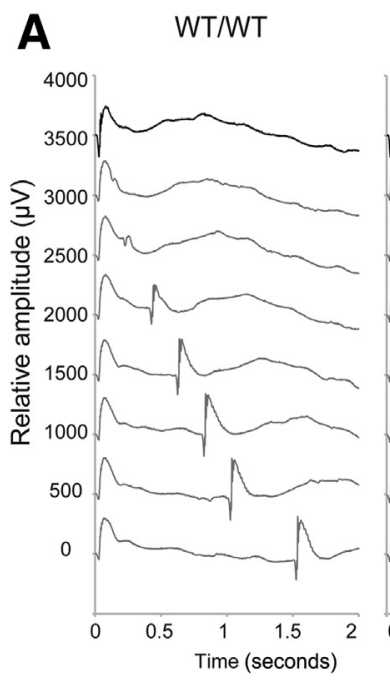

WT/KI

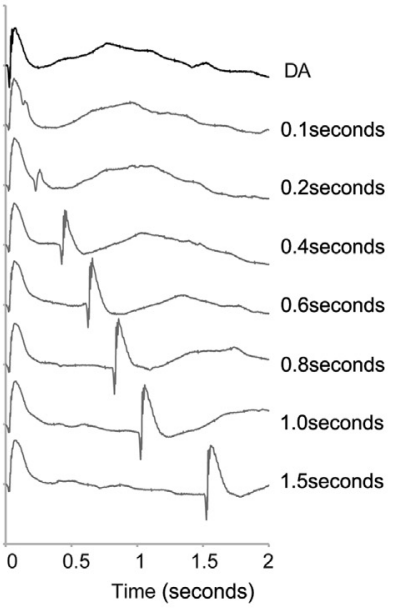

C

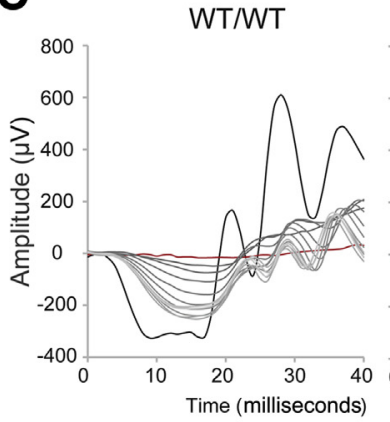

WT/KI

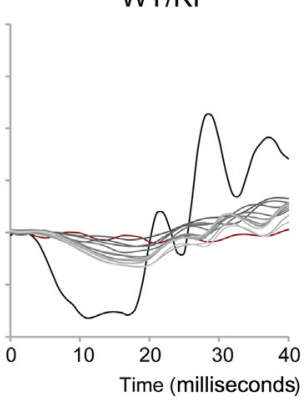

B

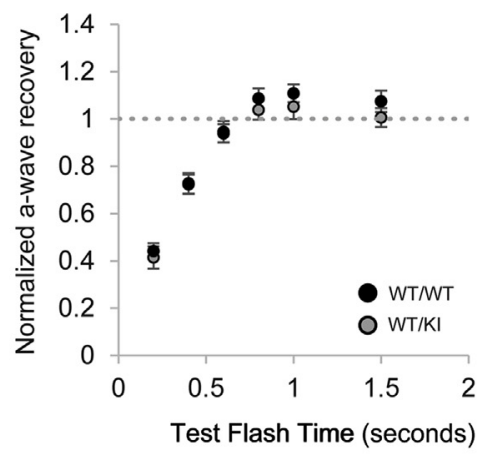

D

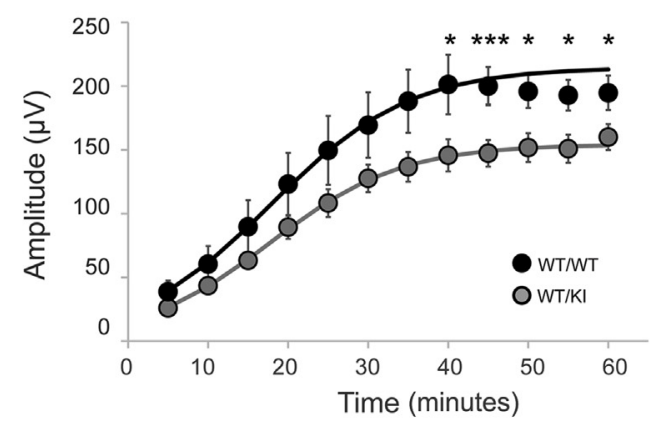

Figure 6 Analysis of visual chromophore regeneration. A: Double-flash electroretinography (ERG) on WT/WT and WT/KI mice. Fully dark-adapted mice were subjected to single flash $\left(4 \mathrm{~cd} \cdot \mathrm{s} / \mathrm{m}^{2}\right)$, followed by test flashes $(40 \mathrm{~cd} \cdot \mathrm{s} / \mathrm{m} 2)$ at various delay times. Representative waveforms from each genotype are shown. B: Normalized amplitude of A-wave from WT/WT and WT/KI mice are shown. A-wave amplitude generated from each test flash was normalized by a fully darkadapted A-wave amplitude. C: Dark-adaptation recovery ERG examining the A-wave value. Mice in indicated groups were bleached at $1000 \mathrm{~cd} / \mathrm{m}^{2} \mathrm{for} 2 \mathrm{minutes,}$ followed by 60 minutes of rest in the dark. A single-flash ERG response at $10 \mathrm{~cd} \cdot \mathrm{s} / \mathrm{m}^{2}$ was acquired every 5 minutes. Representative waveforms from each genotype are shown. Black and red traces are before and after bleach ERG responses, respectively. Response from each time point is depicted in varying shades of gray. D: Averaged recovering A-wave amplitudes of WT/WT and WT/KI mice are shown. Data were fitted using a nonlinear regression curve with previously published formula. ${ }^{32}$ Data are given as means \pm SEM (B and D). $n=6$ (B, WT/WT); $n=8$ (B, WT/KI); $n=10$ (D, WT/WT); $n=16$ (D, WT/KI). ${ }^{*} P<0.05$, $* * * P<0.001$ (t-test). KI, knock in; WT, wild type.

on the visual chromophore regeneration, so that even reduced isomerase activity in the heterozygous $\mathrm{KI}$ mice can sustain appropriate levels of 11-cis-RAL required for normal vision and retinal health. In support of this notion, a recent publication on $P 25 L$ hypomorphic mutant of RPE65 also demonstrated that the homozygous KI mice raised under normal light condition $(<100$ lux $)$ exhibited normal retinal morphology, as well as normal ERGs. ${ }^{32}$ Therefore, it is likely that phenotypes recapitulating the human diseases in some transgenic mouse models may require a brighter
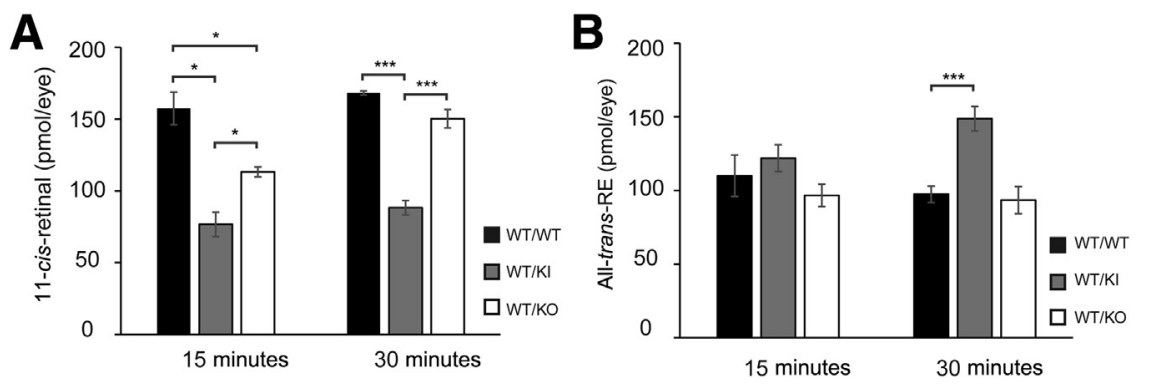

Figure 7 High-performance liquid chromatography (HPLC) analysis on 11-cis-RAL regeneration after photobleaching. HPLC retinoid profile analysis to quantify 11-cis-retinal (A) and all-transretinyl ester (all-trans-RE; B) in WT/WT, WT/KI, and WT/KO mice. Mice were subjected to $30 \mathrm{mi}-$ nutes of bleach in a light box with 5000 lux fluorescent light, followed by recovery in the dark for 15 or 30 minutes. The eyes were dissected and homogenized in the dark for HPLC analysis. Data are given as means $\pm \operatorname{SEM}$ (A and $\mathbf{B}$ ). $n=4$. ${ }^{*} P<0.05,{ }^{* * *} P<0.001$ ( $t$-test). $\mathrm{KI}$, knock in; K0, knockout; WT, wild type. 
lighting condition in husbandry that would be somewhat equivalent to illuminance that humans are daily exposed to. It would be certainly interesting to raise our KI mice under a brighter light condition to determine whether retinal morphology changes, as seen in human patients. In addition, it will be worthwhile to investigate how $D 477 G$ may affect cone function under the optimized stress condition. Because human patients with $D 477 G$ develop ring scotoma in the regions expected to have abundant cone photoreceptors, ${ }^{26,27}$ and depletion of 11-cis-RAL negatively affects the cone photoreceptor health, ${ }^{31}$ the optimized stress condition might lead to retinal morphology changes in our KI mice that resemble human patients. Of interest, slower 11-cis-RAL regeneration rate in $P 25 L \mathrm{KI}$ mice conferred a protective effect against retinal damage induced by high-intensity light (20,000 lux). Taken together, these imply that there may exist a particular window of illuminance in which pathogenic effects of mutant RPE65 can be brought forth.

In conclusion, our KI mouse model of $D 477 G$ partially recapitulates the clinical features reported in the patients with adRP or choroideremia caused by the $D 477 G$ mutation. Future investigation on our KI mouse model with emphasis on various lighting conditions and the mechanism by which the D477G mutant suppresses enzymatic activity of WT RPE65 will be an interesting pursuit, and the information generated may advance our management of retinal dystrophies pertaining to a defective visual cycle.

\section{Acknowledgments}

We thank Dr. Yusuke Takahashi and Dr. Krysten Farjo (The University of Oklahoma Health Sciences Center) for critical discussion and assistance; staff members at the vision core facility (The University of Oklahoma Health Sciences Center); Barb Nagel (St. Louis University) for assisting with transmission electron microscopy; and Dr. Michael Redmond (National Eye Institute) for providing Rpe65 $5^{-1-}$ mice.

\section{References}

1. Wald G: The molecular basis of visual excitation. Nature 1968, 219: 800-807

2. Wald G: The chemistry of rod vision. Science 1951, 113:287-291

3. Heck M, Schädel SA, Maretzki D, Bartl FJ, Ritter E, Palczewski K, Hofmann KP: Signaling states of rhodopsin: formation of the storage form, metarhodopsin III, from active metarhodopsin II. J Biol Chem 2003, 278:3162-3169

4. Saari JC, Bredberg DL: Lecithin:retinol acyltransferase in retinal pigment epithelial microsomes. J Biol Chem 1989, 264:8636-8640

5. Ma J, Zhang J, Othersen KL, Moiseyev G, Ablonczy Z, Redmond TM, Chen Y, Crouch RK: Expression, purification, and MALDI analysis of RPE65. Invest Ophthalmol Vis Sci 2001, 42:1429-1435

6. Pepperberg DR, Okajima TL, Wiggert B, Ripps H, Crouch RK, Chader GJ: Interphotoreceptor retinoid-binding protein (IRBP): molecular biology and physiological role in the visual cycle of rhodopsin. Mol Neurobiol 1993, 7:61-85

7. Kiser PD, Golczak M, Maeda A, Palczewski K: Key enzymes of the retinoid (visual) cycle in vertebrate retina. Biochim Biophys Acta 2012, 1821:137-151
8. Perrault I, Hanein S, Gerber S, Barbet F, Ducroq D, Dollfus H, Hamel C, Dufier J-L, Munnich A, Kaplan J, Rozet J-M: Retinal dehydrogenase 12 (RDH12) mutations in Leber congenital amaurosis. Am J Hum Genet 2004, 75:639-646

9. Rattner A, Smallwood PM, Nathans J: Identification and characterization of all-trans-retinol dehydrogenase from photoreceptor outer segments, the visual cycle enzyme that reduces all-trans-retinal to alltrans-retinol. J Biol Chem 2000, 275:11034-11043

10. Moiseyev G, Crouch RK, Goletz P, Oatis J, Redmond TM, Ma JX: Retinyl esters are the substrate for isomerohydrolase. Biochemistry 2003, 42:2229-2238

11. Takahashi Y, Moiseyev G, Chen Y, Ma JX: Identification of conserved histidines and glutamic acid as key residues for isomerohydrolase activity of RPE65, an enzyme of the visual cycle in the retinal pigment epithelium. FEBS Lett 2005, 579:5414-5418

12. Redmond TM, Yu S, Lee E, Bok D, Hamasaki D, Chen N, Goletz P, Ma JX, Crouch RK, Pfeifer K: Rpe65 is necessary for production of 11-cis-vitamin A in the retinal visual cycle. Nat Genet 1998, 20: 344-351

13. Takahashi Y, Moiseyev G, Nikolaeva O, Ma JX: Identification of the key residues determining the product specificity of isomerohydrolase. Biochemistry 2012, 51:4217-4225

14. Jin M, Li S, Moghrabi WN, Sun H, Travis GH: Rpe65 is the retinoid isomerase in bovine retinal pigment epithelium. Cell 2005, 122: 449-459

15. Farjo KM, Moiseyev G, Takahashi Y, Crouch RK, Ma JX: The 11-cis-retinol dehydrogenase activity of RDH10 and its interaction with visual cycle proteins. Invest Ophthalmol Vis Sci 2009, 50: 5089-5097

16. Kim TS, Maeda A, Maeda T, Heinlein C, Kedishvili N, Palczewski K Nelson PS: Delayed dark adaptation in 11-cis-retinol dehydrogenasedeficient mice: a role of RDH11 in visual processes in vivo. $\mathrm{J}$ Biol Chem 2005, 280:8694-8704

17. Driessen CA, Winkens HJ, Hoffmann K, Kuhlmann LD, Janssen BP, Van Vugt AH, Van Hooser JP, Wieringa BE, Deutman AF, Palczewski K, Ruether K, Janssen JJ: Disruption of the 11-cis-retinol dehydrogenase gene leads to accumulation of cis-retinols and cisretinyl esters. Mol Cell Biol 2000, 20:4275-4287

18. Yamamoto H, Simon A, Eriksson U, Harris E, Berson EL, Dryja TP: Mutations in the gene encoding 11-cis retinol dehydrogenase cause delayed dark adaptation and fundus albipunctatus. Nat Genet 1999, 22: $188-191$

19. Gonzalez-Fernandez F, Kurz D, Bao Y, Newman S, Conway BP, Young JE, Han DP, Khani SC: 11-cis Retinol dehydrogenase mutations as a major cause of the congenital night-blindness disorder known as fundus albipunctatus. Mol Vis 1999, 5:41

20. Thompson DA, Li Y, McHenry CL, Carlson TJ, Ding X, Sieving PA, Apfelstedt-Sylla E, Gal A: Mutations in the gene encoding lecithin retinol acyltransferase are associated with early-onset severe retinal dystrophy. Nat Genet 2001, 28:123-124

21. Marlhens F, Bareil C, Griffoin J-M, Zrenner E, Amalric P, Eliaou C, Liu S-Y, Harris E, Redmond TM, Arnaud B, Claustres M, Hamel CP: Mutations in RPE65 cause Leber's congenital amaurosis. Nat Genet 1997, 17:139-141

22. Gu SM, Thompson DA, Srikumari CR, Lorenz B, Finckh U, Nicoletti A, Murthy KR, Rathmann M, Kumaramanickavel G, Denton MJ, Gal A: Mutations in RPE65 cause autosomal recessive childhood-onset severe retinal dystrophy. Nat Genet 1997, 17:194-197

23. Chacon-Camacho OF, Zenteno JC: Review and update on the molecular basis of Leber congenital amaurosis. World J Clin Cases 2015, 3:112-124

24. Bainbridge JWB, Smith AJ, Barker SS, Robbie S, Henderson R, Balaggan K, Viswanathan A, Holder GE, Stockman A, Tyler N, Petersen-Jones S, Bhattacharya SS, Thrasher AJ, Fitzke FW, Carter BJ, Rubin GS, Moore AT, Ali RR: Effect of gene therapy on visual function in Leber's congenital amaurosis. N Engl J Med 2008, 358: 2231-2239 
25. Morimura H, Fishman GA, Grover SA, Fulton AB, Berson EL, Dryja TP: Mutations in the RPE65 gene in patients with autosomal recessive retinitis pigmentosa or leber congenital amaurosis. Proc Natl Acad Sci U S A 1998, 95:3088-3093

26. Bowne SJ, Humphries MM, Sullivan LS, Kenna PF, Tam LC, Kiang AS, Campbell M, Weinstock GM, Koboldt DC, Ding L, Fulton RS, Sodergren EJ, Allman D, Millington-Ward S, Palfi A, McKee A, Blanton SH, Slifer S, Konidari I, Farrar GJ, Daiger SP, Humphries P: A dominant mutation in RPE65 identified by wholeexome sequencing causes retinitis pigmentosa with choroidal involvement. Eur J Hum Genet 2011, 19:1074-1081

27. Hull S, Mukherjee R, Holder GE, Moore AT, Webster AR: The clinical features of retinal disease due to a dominant mutation in RPE65. Mol Vis 2016, 22:626-635

28. Chakraborty D, Ding X-Q, Conley SM, Fliesler SJ, Naash MI: Differential requirements for retinal degeneration slow intermolecular disulfide-linked oligomerization in rods versus cones. Hum Mol Genet 2009, 18:797-808

29. Farjo R, Skaggs JS, Nagel BA, Quiambao AB, Nash ZA, Fliesler SJ, Naash MI: Retention of function without normal disc morphogenesis occurs in cone but not rod photoreceptors. J Cell Biol 2006, 173:59-68

30. Moiseyev G, Chen Y, Takahashi Y, Wu BX, Ma JX: RPE65 is the isomerohydrolase in the retinoid visual cycle. Proc Natl Acad Sci U S A 2005, 102:12413-12418

31. Rohrer B, Lohr HR, Humphries P, Redmond TM, Seeliger MW, Crouch RK: Cone opsin mislocalization in Rpe65-/- mice: a defect that can be corrected by 11-cis retinal. Invest Ophthalmol Vis Sci 2005, 46: 3876-3882

32. Li Y, Yu S, Duncan T, Li Y, Liu P, Gene E, Cortes-Pena Y, Qian H, Dong L, Redmond TM: Mouse model of human RPE65 P25L hypomorph resembles wild type under normal light rearing but is fully resistant to acute light damage. Hum Mol Genet 2015, 24:4417-4428

33. Samardzija M, Von lintig J, Tanimoto N, Oberhauser V, Thiersch M, Seeliger M, Rem CE, Grimm C, Wenzel A: R91W mutation in Rpe65 leads to milder early-onset retinal dystrophy due to the generation of low levels of 11-cis-retinal. Hum Mol Genet 2008, 17:281-292

34. Cideciyan AV, Aleman TS, Boye SL, Schwartz SB, Kaushal S, Roman AJ, Pang J-J, Sumaroka A, Windsor EAM, Wilson JM, Flotte TR, Fishman GA, Heon E, Stone EM, Byrne BJ, Jacobson SG, Hauswirth WW: Human gene therapy for RPE65 isomerase deficiency activates the retinoid cycle of vision but with slow rod kinetics. Proc Natl Acad Sci U S A 2008, 105:15112-15117

35. Maguire AM, Simonelli F, Pierce EA, Pugh EN, Mingozzi F, Bennicelli J, Banfi S, Marshall KA, Testa F, Surace EM, Rossi S, Lyubarsky A, Arruda VR, Konkle B, Stone E, Sun J, Jacobs J, Dell'Osso L, Hertle R, Ma J, Redmond TM, Zhu X, Hauck B, Zelenaia O, Shindler KS, Maguire MG, Wright JF, Volpe NJ, McDonnell JW, Auricchio A, High KA, Bennett J: Safety and efficacy of gene transfer for Leber's congenital amaurosis. N Engl J Med 2008, 358:2240

36. Simonelli F, Maguire AM, Testa F, Pierce EA, Mingozzi F, Bennicelli JL, Rossi S, Marshall K, Banfi S, Surace EM, Sun J, Redmond TM, Zhu X, Shindler KS, Ying G, Ziviello C, Acerra C, Wright JF, Mcdonnell JW, High KA, Bennett J, Auricchio A: Gene therapy for Leber's congenital amaurosis is safe and effective through 1. 5 years after vector administration. Mol Ther 2009, 18:643-650

37. Poehner WJ, Fossarello M, Rapoport AL, Aleman TS, Cideciyan AV, Jacobson SG, Wright AF, Danciger M, Farber DB: A homozygous deletion in RPE65 in a small Sardinian family with autosomal recessive retinal dystrophy. Mol Vis 2000, 6:192-198 